\title{
Alfredo Ignacio Feria y Velasco. In memoriam
}

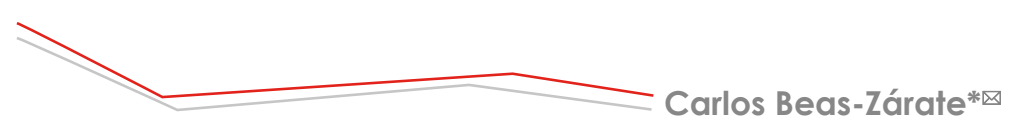

Beas-Zárate, C. (2019). Alfredo Ignacio Feria y Velasco. In memoriam [Obituario]. Investigación y Ciencia de la Universidad Autónoma de Aguascalientes, 27(76), 93-94.

El Dr. Alfredo Ignacio Feria y Velasco, hijo del Sr. Alfredo Feria Feria y de la Sra. Rosa Velasco Pantoja nació en el Distrito Federal, México, el 13 de agosto de 1940 y falleció el 1 de agosto de 2017 en Guadalajara, Jalisco, México. Realizó sus estudios básicos en el Colegio Francés Hidalgo. Médico Cirujano egresado de la Facultad de Medicina de la Universidad Nacional Autónoma de México con especialidad en Anatomía Patológica en el Centro Médico Nacional del Instituto Mexicano del Seguro Social, en el Hospital Penrose en Colorado Springs, CO, EE. UU. y el Hospital Montefiore-Morrisania de Nueva York, NY, EE. UU.

Este destacado científico realizó sus estudios de maestría en Neuroquímica en la Facultad de Química de la UNAM y de doctorado en Neurociencias en el Centro de Investigación y de Estudios Avanzados del Instituto Politécnico Nacional, efectuó estudios de posdoctorado en Neuropatología Experimental en la Escuela de Medicina de la Universidad de Harvard, en Boston, MA, EE. UU.

Incansable académico, desarrolló líneas de investigación sobre el desarrollo del sistema nervioso central y bases morfofisiológicas de los mecanismos de producción de convulsiones. Durante su prolífera vidacomo investigadorpublicó 211 trabajosenrevistas científicas arbitradas con circulación internacional, 247 comunicaciones breves, 80 capítulos en libros y es autor o coautor de cinco libros en su área de especialidad. El Dr. Feria y Velasco se desempeñó como docente de licenciatura y posgrado, dirigió 14 tesis de licenciatura, 21 de maestría en Ciencias y 16 de doctorado en Ciencias.

\footnotetext{
* Departamento de Biología Celular y Molecular, Centro Universitario de Ciencias Biológicas y Agropecuarias, Universidad de Guadalajara. Camino Ramón Padilla Sánchez 2100, Nextipac, C. P. 44600, Zapopan, Jalisco, México. Correo electrónico: carlos.beas@academicos.udg. mx. ORCID: orcid.org/0000-0002-2922-2868

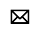

Autor para correspondencia
}

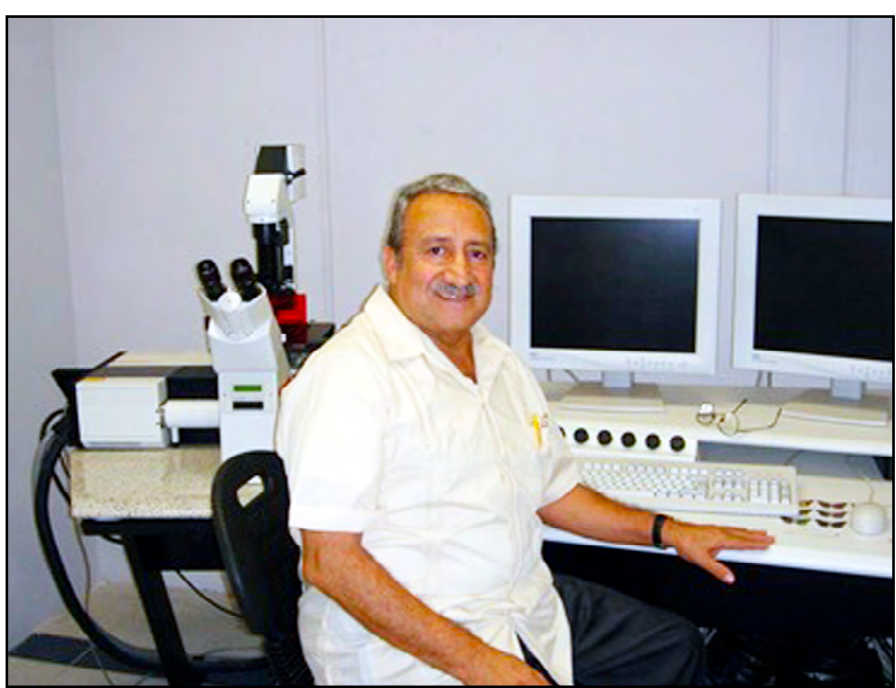

Dr. Alfredo Feria y Velasco.

Fotografía proporcionada en 2016 por el Dr. Feria y Velasco al autor del presente.

Por su notorio trabajo científico y su participación en la formación de recursos humanos para la investigación, ingresó al Sistema Nacional de Investigadores (SNI) en 1984 como nivel II, fue en tres ocasiones consecutivas Investigador Nacional nivel III y en enero de 2008 fue reconocido como Investigador Nacional Emérito.

En su trayectoria logró formar investigadores que desarrollaron laboratorios de investigación en todo el occidente del país, en particular en los estados de Jalisco, Michoacán, Colima, Aguascalientes, Durango y Nayarit. Varios de sus estudiantes han logrado ser miembros del SNI en todos los niveles y con posiciones académico-administrativas importantes en diversas universidades.

Se destacó no solamente como científico, sino como excelente gestor, así fue director de la Unidad de Investigación Biomédica de Occidente del IMSS, titular de la subjefatura de Investigación Biomédica 
del mismo instituto a nivel nacional, investigador y coordinador académico del Centro de Investigación y Asistencia en Tecnología y Diseño del Estado de Jalisco, director de la División de Ciencias Biológicas y Ambientales del Centro Universitario de Ciencias Biológicas y Agropecuarias (CUCBA) de la Universidad de Guadalajara, coordinador de Investigación del CUCBA, y coordinador de Investigación y Posgrado de la Universidad de Guadalajara. Director del Centro de Instrumentación Transdisciplinaria y de Servicios (CITRANS, hoy ITRANS) de la Universidad de Guadalajara.

Su genuina vocación científica lo llevó a trabajar hasta el final en el estudio de las bases morfológicas del desarrollo del sistema nervioso central y los mecanismos de producción de convulsiones y excitotoxicidad, como jefe del laboratorio de Neurobiología Celular del Departamento de Biología Celular y Molecular en el Centro Universitario de Ciencias Biológicas y Agropecuarias de la Universidad de Guadalajara.

Fue un gran académico, amigo, hombre probo de profundas conversaciones, cuyos dichos y chistes hacían su compañía entrañable, que degustaba una comida con buen vino sin faltar el Cardenal de Mendoza como digestivo; igual disfrutaba el momento con un estudiante que con un gran investigador, político o administrativo de alto nivel $y$, en todo proyecto a iniciar, al final siempre utilizó su frase célebre: "Hay que echarle cerebro". 